International Journal of Applied Mathematical Research, 1 (2) (2012) 141-149

\title{
Soliton Solutions for (2+1)-Dimensional Breaking Soliton Equation: Three Wave Method
}

\author{
Somayeh Arbabi Mohammad Abadi, Maliheh Najafi \\ Department of Mathematics, Anar Branch, Islamic Azad University, Anar, Iran \\ Email:arbabi_math87@yahoo.com \\ Email:malihe_math87@yahoo.com
}

\begin{abstract}
By means of the three-wave method one can solve some nonlinear partial differential equations (NLPDEs) in their bilinear forms. When an NLPDE has no bilinear closed form we can not use this method. We modify the idea of three-wave method to obtain some analytic solutions for the $(2+1)$-dimensional Breaking soliton equation by obtaining a bilinear closed form for it. By comparison of this method and other analytic methods, like HAM, HTA and EHTA, we can see that the new idea is very easy and straightforward.
\end{abstract}

Keywords: Three-wave method; BS equation; Hirota's bilinear form.

\section{Introduction}

Many important phenomena and dynamic processes in physics, mechanics, chemistry and biology can be represented by nonlinear partial differential equations. The study of exact solutions of nonlinear evolution equations plays an important role in soliton theory and explicit formulas of nonlinear partial differential equations play an essential role in the nonlinear science. Also, the explicit formulas may provide physical information and help us to understand the mechanism of related physical models. Recently, many kinds of powerful methods have been proposed to find exact solutions of nonlinear partial differential 
equations, e.g., the tanh-method [1], the homogeneous balance method [2], homotopy analysis method $[3,4,5,6,7,8]$, the $F$-expansion method [9], threewave method $[10,11,12]$, extended homoclinic test approach $[13,14,15]$, the $\left(\frac{G^{\prime}}{G}\right)$-expansion method [16] and the exp-function method [17, 18, 19, 20, 21].

Dai et al. [22], suggested the three-wave method for nonlinear evolution equations. The basic idea of this method applies the Painlevé analysis to make a transformation as

$$
u=T(f)
$$

for some new and unknown function $f$.Then we use this transformation in a high dimensional nonlinear equation of the general form

$$
F\left(u, u_{t}, u_{x}, u_{y}, u_{z}, u_{x x}, u_{y y}, u_{z z}, \cdots\right)=0,
$$

where $u=u(x, y, z, t)$ and $F$ is a polynomial of $u$ and its derivatives. By substituting (1) in (2), the first one converts into the Hirota's bilinear form, which it will solve by taking a special form for $f$ and assuming that the obtained Hirota's bilinear form has three-wave solutions, we can specify the unknown function $f$. For more details see $[22,23]$.

\section{Soliton Solutions to the $(2+1)$-dimensional Breaking Soliton equation}

In this paper, we investigate explicit formula of solutions of the following $(2+1)$-Dimensional Breaking Soliton equation given in [24]

$$
u_{x x x y}-2 u_{y} u_{x x}-4 u_{x} u_{x y}+u_{x t}=0 .
$$

To solve eq. (3) authors in [24] used of N-soliton solution. In this paper, we use the idea of three-wave method $[22,23]$, to solve equation $(3)$. By this idea we obtain some analytic solutions for the problem. The process of our method is very easy and more simple than the method of Ting et al. [24]. To solve eq. (3), we introduce a new dependent variable $w$ by

$$
w=-2(\ln f)_{x}
$$

where $f(x, y, t)$ is an unknown real function which will be determined. Substituting eq. (4) into eq. (3), we have

$$
2(\ln f)_{x x t}+2(\ln f) x x x x y+16(\ln f)_{x x}(\ln f)_{x x y}+8(\ln f)_{x x x}(\ln f)_{x y}=0,
$$

which can be integrated once with respect to $x$ to give

$$
\begin{aligned}
& 2(\ln f)_{x t}+2(\ln f)_{x x x y}+12(\ln f)_{x x}(\ln f)_{x y} \\
+ & 4 \partial_{x}^{-1}\left((\ln f)_{x x}(\ln f)_{x x y}-(\ln f)_{x x x}(\ln f)_{x y}\right)=0,
\end{aligned}
$$


Therefore, eq. (6) can be written as

$$
\left(D_{x} D_{t}+D_{y} D_{x}^{3}\right) f \cdot f+4 f^{2} \partial_{x}^{-1}\left(D_{x}(\ln f)_{x x} \cdot(\ln f)_{x y}\right)=0,
$$

where the D-operator is defined by

$$
\begin{aligned}
& D_{x}^{m} D_{t}^{n} f(x, t) \cdot g(x, t)= \\
& \left.\quad\left(\frac{\partial}{\partial x_{1}}-\frac{\partial}{\partial x_{2}}\right)^{m}\left(\frac{\partial}{\partial t_{1}}-\frac{\partial}{\partial t_{2}}\right)^{n}\left[f\left(x_{1}, t_{1}\right) g\left(x_{2}, t_{2}\right)\right]\right|_{x_{1}=x_{2}=x, t_{1}=t_{2}=t} .
\end{aligned}
$$

We suppose that

$$
\partial_{x}^{-1}\left(D_{x}(\ln f)_{x x} \cdot(\ln f)_{x y}\right)=0,
$$

then eq. (7) reduces to

$$
\left(D_{x} D_{t}+D_{y} D_{x}^{3}\right) f \cdot f=0,
$$

Now we suppose the solution of eq. (8) as

$$
f(x, y, t)=\mathrm{e}^{-\xi_{1}}+\delta_{1} \cos \left(\xi_{2}\right)+\delta_{2} \cosh \left(\xi_{3}\right)+\delta_{3} \mathrm{e}^{\xi_{1}}
$$

where

$$
\xi_{i}=a_{i} x+b_{i} y+c_{i} t, \quad i=1,2,3
$$

and $a_{i}, c_{i}, \delta_{i}$ are some constants to be determined later. Substituting eq. (9) into eq. (8), and equating all the coefficients of $\sin \left(a_{2} x+b_{2} y+c_{2} t\right)$, $\cos \left(a_{2} x+b_{2} y+c_{2} t\right), \sinh \left(a_{3} x+b_{3} y+c_{3} t\right)$ and $\cosh \left(a_{3} x+b_{3} y+c_{3} t\right)$ to zero, we get the set of algebraic equation for $a_{i}, b_{i}, c_{i}, \delta_{i}, \quad(i=1,2,3)$

$$
\begin{aligned}
& -3 a_{1}^{2} b_{1} a_{3}-a_{1}^{3} b_{3}-3 b_{3} a_{3}^{2} a_{1}-a_{3}^{3} b_{1}-c_{3} a_{1}-c_{1} a_{3}=0, \\
& 3 a_{1} b_{1} a_{3}^{2}+c_{1} a_{1}+a_{3} c_{3}+a_{1}^{3} b_{1}+b_{3} a_{3}^{3}+3 b_{3} a_{3} a_{1}^{2}=0, \\
& -a_{2} c_{2}+b_{2} a_{2}^{3}+a_{1}^{3} b_{1}+c_{1} a_{1}-3 b_{2} a_{2} a_{1}^{2}-3 a_{1} b_{1} a_{2}^{2}=0, \\
& a_{1}^{3} b_{2}+c_{2} a_{1}+3 a_{1}^{2} b_{1} a_{2}+c_{1} a_{2}-a_{2}^{3} b_{1}-3 b_{2} a_{2}^{2} a_{1}=0, \\
& -a_{2}{ }^{3} b_{3}+c_{2} a_{3}+c_{3} a_{2}+a_{3}^{3} b_{2}-3 b_{2} a_{2}^{2} a_{3}+3 b_{3} a_{3}^{2} a_{2}=0, \\
& a_{3} c_{3}+b_{3} a_{3}^{3}-a_{2} c_{2}-3 b_{3} a_{3} a_{2}^{2}+b_{2} a_{2}^{3}-3 b_{2} a_{2} a_{3}^{2}=0, \\
& 16 a_{1}^{3} b_{1} \delta_{3}+4 c_{1} a_{1} \delta_{3}-\delta_{1}{ }^{2} c_{2} a_{2}+\delta_{2}{ }^{2} c_{3} a_{3}+4 \delta_{1}{ }^{2} a_{2}{ }^{3} b_{2}+4 \delta_{2}{ }^{2} a_{3}{ }^{3} b_{3}=0
\end{aligned}
$$

Solving the system of equations (11) with the aid of Maple, we obtain the following cases: 


\subsection{CaseI:}

$$
\begin{aligned}
& a_{1}=a_{3}, a_{2}=0, b_{1}=-b_{3}, b_{3}=-\frac{c_{3}}{a_{3}^{2}}, \\
& c_{1}=-c_{3}, c_{2}=-a_{3}^{2} b_{2}, \delta_{1}=0, \delta_{3}=\frac{\delta_{2}^{2}}{4},
\end{aligned}
$$

for some arbitrary real constants $a_{3}, c_{3}, b_{2}$ and $\delta_{2}$. Substitute eq. (12) into eq. (4) with eq. (9), we obtain the solution as

$$
f(x, y, t)=\mathrm{e}^{-\xi_{1}}+\delta_{2} \cosh \left(\xi_{2}\right)+\delta_{3} \mathrm{e}^{\xi_{1}}
$$

and

$$
u(x, y, t)=\frac{-2\left(-a_{3} \mathrm{e}^{-\xi_{1}}+\delta_{2} \sinh \left(\xi_{2}\right) a_{3}+\delta_{3} a_{3} \mathrm{e}^{\xi_{1}}\right)}{\mathrm{e}^{-\xi_{1}}+\delta_{2} \cosh \left(\xi_{2}\right)+\delta_{3} \mathrm{e}^{\xi_{1}}}
$$

for

$$
\xi_{1}=a_{3} x-b_{3} y-c_{3} t \quad, \quad \xi_{2}=a_{3} x-\frac{c_{3}}{a_{3}^{2}} y+c_{3} t \quad, \quad \delta_{3}=\frac{1}{4} \delta_{2}^{2}
$$

If $\delta_{3}>0$, then we obtain the exact breather cross-kink solution

$$
u(x, y, t)=\frac{-2 a_{3}\left(2 \sqrt{\delta_{3}} \sinh \left(\xi_{1}-\theta\right)+\delta_{2} \sinh \left(\xi_{2}\right)\right)}{2 \sqrt{\delta_{3}} \cosh \left(\xi_{1}-\theta\right)+\delta_{2} \cosh \left(\xi_{2}\right)}
$$

for

$$
\theta=\frac{1}{2} \ln \left(\delta_{3}\right) \quad, \quad \delta_{3}=\frac{1}{4} \delta_{2}^{2}
$$

If $\delta_{3}<0$, then we obtain the exact breather cross-kink solution

$$
u(x, y, t)=-2 \frac{a_{3}\left(2 \sqrt{-\delta_{3}} \cosh \left(\xi_{1}-\theta\right)+\delta_{2} \sinh \left(\xi_{2}\right)\right)}{2 \sqrt{-\delta_{3}} \sinh \left(\xi_{1}-\theta\right)+\delta_{2} \cosh \left(\xi_{2}\right)}
$$

for

$$
\theta=\frac{1}{2} \ln \left(-\delta_{3}\right) \quad, \quad \delta_{3}=\frac{1}{4} \delta_{2}^{2}
$$

\subsection{CaseII:}

$$
\begin{gathered}
a_{1}=a_{3}, b_{1}=b_{3}, c_{1}=c_{3}=-4 b_{3} a_{3}^{2}, \delta_{1}=0 \\
c_{2}=-\frac{1}{2} \frac{b_{3}\left(a_{2}^{4}+6 a_{3}^{2} a_{2}^{2}-3 a_{3}^{4}\right)}{a_{2} a_{3}}, b_{2}=-\frac{1}{2} \frac{b_{3}\left(a_{2}^{2}+3 a_{3}^{2}\right)}{a_{2} a_{3}}
\end{gathered}
$$


for some arbitrary real constants $a_{3}, a_{2}, b_{3}, \delta_{i}, i=1,2$. Substitute eq. (14) into eq. (4) with eq. (9), we obtain the solution as follows

$$
f(x, y, t)=\mathrm{e}^{-\xi_{1}}+\delta_{2} \cosh \left(\xi_{1}\right)+\delta_{3} \mathrm{e}^{\xi_{1}}
$$

and

$$
u(x, y, t)=\frac{-2\left(-a_{3} \mathrm{e}^{-\xi_{1}}+\delta_{2} \sinh \left(\xi_{1}\right) a_{3}+\delta_{3} a_{3} \mathrm{e}^{\xi_{1}}\right)}{\mathrm{e}^{-\xi_{1}}+\delta_{2} \cosh \left(\xi_{1}\right)+\delta_{3} \mathrm{e}^{\xi_{1}}}
$$

for

$$
\xi_{1}=a_{3} x+b_{3} y-4 b_{3} a_{3}{ }^{2} t
$$

If $\delta_{3}>0$ then we obtain the exact breather cross-kink solution

$$
u(x, y, t)=\frac{-2 a_{3}\left(2 \sqrt{\delta_{3}} \sinh \left(\xi_{1}-\theta\right)+\delta_{2} \sinh \left(\xi_{1}\right)\right)}{2 \sqrt{\delta_{3}} \cosh \left(\xi_{1}-\theta\right)+\delta_{2} \cosh \left(\xi_{1}\right)}
$$

for

$$
\theta=\frac{1}{2} \ln \left(\delta_{3}\right)
$$

If $\delta_{3}<0$ then we obtain the exact breather cross-kink solution

$$
u(x, y, t)=\frac{-2 a_{3}\left(2 \sqrt{-\delta_{3}} \cosh \left(\xi_{1}-\theta\right)+\delta_{2} \sinh \left(\xi_{1}\right)\right)}{-2 \sqrt{-\delta_{3}} \sinh \left(\xi_{1}-\theta\right)+\delta_{2} \cosh \left(\xi_{1}\right)}
$$

for

$$
\theta=\frac{1}{2} \ln \left(-\delta_{3}\right)
$$

\subsection{CaseIII:}

$$
\begin{aligned}
& a_{1}=a_{3}, a_{2}=0, b_{1}=-b_{3}, b_{3}=-\frac{c_{3}}{a_{3}^{2}}, \\
& c_{1}=-c_{3}, c_{2}=-a_{3}^{2} b_{2}, \delta_{1}=0, \delta_{3}=\frac{\delta_{2}^{2}}{4},
\end{aligned}
$$

for some arbitrary real constants $a_{3}, c_{3}, b_{2}$ and $\delta_{2}$. Substitute eq. (16) into eq. (4) with eq. (9), we obtain the solution as

$$
f(x, y, t)=\mathrm{e}^{-\xi_{1}}+\delta_{2} \cosh \left(\xi_{2}\right)+\delta_{3} \mathrm{e}^{\xi_{1}}
$$

and

$$
u(x, y, t)=\frac{3}{2} \frac{-a_{3} \mathrm{e}^{-\xi_{1}}+\delta_{2} \cosh \left(\xi_{2}\right) a_{3}+\delta_{3} a_{3} \mathrm{e}^{\xi_{1}}}{\mathrm{e}^{-\xi_{1}}+\delta_{2} \sinh \left(\xi_{2}\right)+\delta_{3} \mathrm{e}^{\xi_{1}}}
$$


for

$$
\xi_{1}=a_{3} x-b_{3} y-c_{3} t \quad, \quad \xi_{2}=a_{3} x-\frac{c_{3}}{a_{3}^{2}} y+c_{3} t \quad, \quad \delta_{3}=\frac{1}{4} \delta_{2}^{2}
$$

If $\delta_{3}>0$, then we obtain the exact breather cross-kink solution

$$
u(x, y, t)=\frac{3}{2} \frac{a_{3}\left(2 \sqrt{\delta_{3}} \cosh \left(\xi_{1}-\theta\right)+\delta_{2} \cosh \left(\xi_{2}\right)\right)}{2 \sqrt{\delta_{3}} \sinh \left(\xi_{1}-\theta\right)+\delta_{2} \sinh \left(\xi_{2}\right)}
$$

for

$$
\theta=\frac{1}{2} \ln \left(\delta_{3}\right) \quad, \quad \delta_{3}=\frac{1}{4} \delta_{2}^{2}
$$

If $\delta_{3}<0$, then we obtain the exact breather cross-kink solution

$$
u(x, y, t)=\frac{3}{2} \frac{a_{3}\left(2 \sqrt{-\delta_{3}} \sinh \left(\xi_{1}-\theta\right)+\delta_{2} \cosh \left(\xi_{2}\right)\right)}{2 \sqrt{-\delta_{3}} \cosh \left(\xi_{1}-\theta\right)+\delta_{2} \sinh \left(\xi_{2}\right)}
$$

for

$$
\theta=\frac{1}{2} \ln \left(-\delta_{3}\right) \quad, \quad \delta_{3}=\frac{1}{4} \delta_{2}^{2}
$$

\subsection{CaseIV:}

$$
\begin{gathered}
a_{1}=a_{3}, b_{1}=b_{3}, c_{1}=c_{3}=-4 b_{3} a_{3}^{2}, \delta_{1}=0 \\
c_{2}=-\frac{1}{2} \frac{b_{3}\left(a_{2}^{4}+6 a_{3}^{2} a_{2}^{2}-3 a_{3}^{4}\right)}{a_{2} a_{3}}, b_{2}=-\frac{1}{2} \frac{b_{3}\left(a_{2}{ }^{2}+3 a_{3}{ }^{2}\right)}{a_{2} a_{3}}
\end{gathered}
$$

for some arbitrary real constants $a_{3}, a_{2}, b_{3}, \delta_{i}, i=1,2$. Substitute eq. (18) into eq. (4) with eq. (9), we obtain the solution as follows

$$
f(x, y, t)=\mathrm{e}^{-\xi_{1}}+\delta_{2} \cosh \left(\xi_{1}\right)+\delta_{3} \mathrm{e}^{\xi_{1}}
$$

and

$$
u(x, y, t)=\frac{3}{2} \frac{-a_{3} \mathrm{e}^{-\xi_{1}}+\delta_{2} \cosh \left(\xi_{1}\right) a_{3}+\delta_{3} a_{3} \mathrm{e}^{\xi_{1}}}{\mathrm{e}^{-\xi_{1}}+\delta_{2} \sinh \left(\xi_{1}\right)+\delta_{3} \mathrm{e}^{\xi_{1}}}
$$

for

$$
\xi_{1}=a_{3} x+b_{3} y-4 b_{3} a_{3}^{2} t
$$

If $\delta_{3}>0$ then we obtain the exact breather cross-kink solution

$$
u(x, y, t)=\frac{3}{2} \frac{a_{3}\left(2 \sqrt{\delta_{3}} \cosh \left(\xi_{1}-\theta\right)+\delta_{2} \cosh \left(\xi_{1}\right)\right)}{2 \sqrt{\delta_{3}} \sinh \left(\xi_{1}-\theta\right)+\delta_{2} \sinh \left(\xi_{1}\right)}
$$


for

$$
\theta=\frac{1}{2} \ln \left(\delta_{3}\right)
$$

If $\delta_{3}<0$ then we obtain the exact breather cross-kink solution

$$
u(x, y, t)=\frac{3}{2} \frac{a_{3}\left(2 \sqrt{-\delta_{3}} \sinh \left(\xi_{1}-\theta\right)+\delta_{2} \cosh \left(\xi_{1}\right)\right)}{-2 \sqrt{-\delta_{3}} \cosh \left(\xi_{1}-\theta\right)+\delta_{2} \sinh \left(\xi_{1}\right)}
$$

for

$$
\theta=\frac{1}{2} \ln \left(-\delta_{3}\right)
$$

\section{Conclusion}

In this paper, using the three-wave solution method we obtained some explicit formulas of solutions for the $(3+1)$-dimensional Soliton equation. Three-wave solution method with the aid of a symbolic computation software like Maple or Mathematica is an easy and straightforward method which can be apply

to other nonlinear partial differential equations. It must be noted that, all obtained solutions have checked in the $(3+1)$-dimensional Soliton equation. All solutions satisfy in the equations.

\section{References}

[1] A.M. Wazwaz,The tanh method: solitons and periodic solutions for the Dodd-Bullough-Tzikhailov and the Tzitzeica-Dodd-Bullough equations, Chaos, Solitons and Fractals, 25 (2005) 55-63.

[2] Z. Xiqiang, W. Limin, S. Weijun, The repeated homogeneous balance method and its applications to nonlinear partial differential equations, Chaos, Solitons and Fractals, 28(2) (2006) 448-453.

[3] S.J. Liao, Beyond Perturbation: Introduction to the homotopy analysis method, Chapman \& Hall/CRC Press, Boca Raton, (2003).

[4] S.J. Liao, On the homotopy analysis method for nonlinear problems, Appl. Math. Comput., 147 (2006) 499-513.

[5] S.J. Liao, A general approach to get series solution of nonsimilarity boundary-layer flows, Commun. Nonlinear Sci. Numer. Simul., 14(5)(2009) 2144-2159.

[6] M.T. Darvishi, F. Khani, A series solution of the foam drainage equation, Comput. Math. Appl., 58 (2009) 360-368. 
[7] A. Aziz, F. Khani, M.T. Darvishi, Homotopy analysis method for variable thermal conductivity heat flux gage with edge contact resistance, Zeitschrift fuer Naturforschung A, 65a(10)(2010) 771-776.

[8] F. Khani, M.T. Darvishi, R.S.R. Gorla, Analytical investigation for cooling turbine disks with a non-Newtonian viscoelastic fluid, Comput. Math. Appl., 61(7)(2011) 1728-1738.

[9] E. Fan, Z. Jian, Applications of the Jacobi elliptic function method to special-type nonlinear equations, Phys. Lett. A, 305(6) (2002) 383-392.

[10] M.T. Darvishi, Maliheh Najafi, Mohammad Najafi, Exact three-wave solutions for high nonlinear form of Benjamin-Bona-Mahony-Burgers equations, International Journal of Mathematical and Computer Sciences, 6(3)(2010) 127-131.

[11] M.T. Darvishi, M. Najafi, Some exact solutions of the $(2+1)$-dimensional breaking soliton equation using the three-wave method, International Journal of Computational and Mathematical Sciences, 6(1)(2012)13-16.

[12] M.T. Darvishi, Maliheh Najafi, Mohammad Najafi, New exact solutions for the $(3+1)$-dimensional breaking soliton equation, International Journal of Information and Mathematical Sciences, 6(2)(2010) 134-137.

[13] M.T. Darvishi, Maliheh Najafi, Mohammad Najafi, New application of EHTA for the generalized $(2+1)$-dimensional nonlinear evolution equations, International Journal of Mathematical and Computer Sciences, 6(3)(2010) 132-138.

[14] M.T. Darvishi, M. Najafi, A modification of extended homoclinic test approach to solve the $(3+1)$-dimensional potential-YTSF equation, Chin. Phys. Lett., 28(4)(2011) 040202.

[15] M.T. Darvishi, M. Najafi, Some complexiton type solutions of the $(3+1)-$ dimensional Jimbo-Miwa equation, International Journal of Computational and Mathematical Sciences, 6(1)(2012) 25-27.

[16] M.T. Darvishi, Maliheh Najafi, Mohammad Najafi, Traveling wave solutions for the $(3+1)$-dimensional breaking soliton equation by $\left(\frac{G^{\prime}}{G}\right)$ expansion method and modified $F$-expansion method, International Journal of Computational and Mathematical Sciences, 6(2)(2012) 64-69.

[17] J.H. He, M.A. Abdou, New periodic solutions for nonlinear evolution equations using Exp-function method, Chaos, Solitons and Fractals, 34 (2007) 1421-1429. 
[18] F. Khani, S. Hamedi-Nezhad, M.T. Darvishi, S.-W. Ryu, New solitary wave and periodic solutions of the foam drainage equation using the Expfunction method, Nonlin. Anal.: Real World Appl., 10 (2009) 1904-1911.

[19] B.-C. Shin, M.T. Darvishi, A. Barati, Some exact and new solutions of the Nizhnik-Novikov-Vesselov equation using the Exp-function method, Comput. Math. Appl., 58(11/12) (2009) 2147-2151.

[20] F. Khani, M.T. Darvishi, A. Farmani, L. Kavitha, New exact solutions of coupled $(2+1)$-dimensional nonlinear system of Schrödinger equations, ANZIAM Journal, 52 (2010) 110-121.

[21] X.H. Wu, J.H. He, Exp-function method and its application to nonlinear equations, Chaos, Solitons and Fractals, 38(3)(2008) 903-910.

[22] Z.-D. Dai, S.-Q. Lin, D.-L. Li, G. Mu, The three-wave method for nonlinear evalution equations, Nonl. Sci. Lett. A, 1(1) (2010)77-82.

[23] C.-J. Wang, Z.-D. Dai, L. Liang, Exact three-wave solution for higher dimensional KDV-type equation, Appl. Math. Comput., 216 (2010.) 501505 .

[24] S. Ting, G.X. Guo, M.Y. Ling, Wronskian form of N-Soliton solution for the (2+1)-dimensional breaking soliton equation, Chin. Phys. Lett., 24(2) (2007) 305-307. 